\title{
Schlechte Prognose trotz Einsatz von Prothrombinkomplex-Konzentrat
}

Fragestellung: Wie ist die Prognose von Warfarin induzierten intrakraniellen Blutungen bei Therapie mit Prothrombinkomplex-Konzentraten?

Hintergrund: Antikoagulanzien-induzierte intrakranielle Blutungen sind größer als spontane intrakranielle Blutungen, haben ein höheres Risiko einer Zunahme des Blutungsvolumens sowie eine schlechtere Prognose. Die meisten Leitlinien empfehlen den Einsatz von entweder Fresh Frozen Plasma oder Prothrombinkomplex-Konzentraten (PCC). Allerdings gibt es nur sehr wenig prospektiv gesammelte Daten zum Einsatz der beiden Therapiemethoden.

Patienten und Methodik: Es handelt sich um ein prospektives multizentrisches Register in Kanada, in welches Patienten eingeschlossen wurden, bei denen eine intrakranielle Blutung mit Prothrombinkomplex-Konzentraten behandelt wurde. Die Patienten wurden über die Blutbanken der entsprechenden teilnehmenden Krankenhäuser identifiziert. Erhoben wurden die klinischen Parameter, Ergebnisse der zerebralen Bildgebung, Laborwerte und thrombotische Ereignisse unter und nach der Therapie. Das Volumen der intrakraniellen Blutungen wurde mithilfe von CT-Aufnahmen gemessen.

Der Outcome wurde bei der Entlassung mithilfe der modifizierten Rankin-Skala bestimmt und außerdem wurde die Sterblichkeit während des Krankenhausaufenthaltes erfasst.

Ergebnisse: Die insgesamt 141 Patienten waren im Mittel 78 Jahre alt. $60 \%$ der Patienten waren Männer. 20\% der Patienten nahmen zum Zeitpunkt der Blutung zusätzlich zu Warfarin Thrombozytenfunktionshemmer ein. Bei rund drei Viertel der Patienten war die Indikation für die Einnahme von Warfarin Vorhofflimmern. Die zweithäufigste Indikation waren tiefe Beinvenenthrombosen. Bei 50\% der intrakraniellen Blutungen handelte es sich um intraparenchymatöse Hirnblutungen, bei $43 \%$ um Subduralhämatome und bei $6 \%$ um Subarachnoidalblutungen. Bei $20 \%$ der Patienten lag eine intraventrikuläre Blutung vor.

Nach der Behandlung mit Prothrombinkomplex-Konzentrat fiel die INR (International Normalized Ratio) von 2,6 auf 1,4 ab. Bei $80 \%$ der Patienten hatte sich die INR innerhalb von einer Stunde nach der Gabe von Prothrombinkomplex-KonzenAsdaghi N; on behalf of the Canadian PCC Registry (CanPro) Investigators. Poor prognosis in Warfarin-associated intracranial hemorrhage despite anticoagulation reversal. Stroke 2012; 43: 1812-7
Dowlatshahi D, Butcher KS, trat normalisiert. Patienten mit intraparenchymatösen Hirnblutungen hatten eine Sterblichkeit von $42,3 \%$. Bei $50 \%$ der Patienten kam es zu einer Ausdehnung des initialen Hämatoms. Innerhalb der ersten sieben Tage nach der Therapie mit Prothrom-

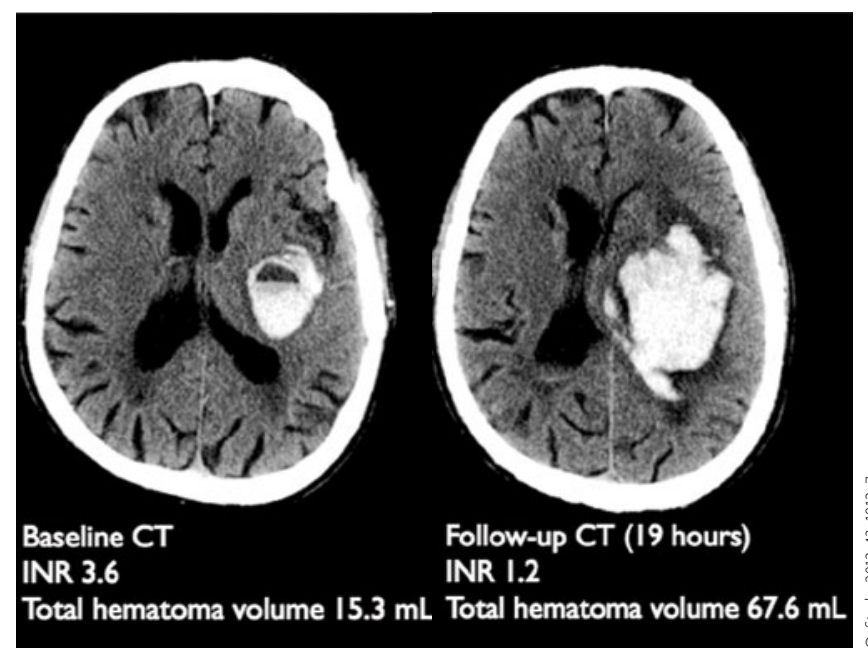

1 Signifikantes Hämatomwachstum trotz INR-Korrektur mit Prothrombinkomplex-Konzentrat.

binkomplex-Konzentraten kam es zu drei thrombotischen Komplikationen.

Schlussfolgerungen: Die Gabe von ProthrombinkomplexKonzentraten bei Warfarin-induzierten intrakraniellen Blutungen normalisiert innerhalb kurzer Zeit die INR, hat aber wahrscheinlich keinen Einfluss auf den klinischen Outcome.

\section{- Kommentar von Hans-Christoph Diener, Essen}

\section{Hohe Wirksamkeit}

Diese Beobachtungsstudie aus Kanada legt nahe, dass Prothrombinkomplex-Konzentrate sehr wirksam sind, um innerhalb kurzer Zeit eine erhöhte INR zu normalisieren. Unabhängig davon, zeigt die hier vorgelegte Studie aber eine Mortalität von $42 \%$. Dies entspricht ziemlich genau der Sterblichkeit die bei intrakraniellen Blutungen in der RELY-Studie beobachtet wurde [1]. In dieser Studie wurden ProthrombinkomplexKonzentrat sehr selten eingesetzt, sodass zu vermuten ist, das Prothrombinkomplex-Konzentrat die Prognose intrakranieller Blutungen unter der Gabe von Antikoagulanzien nicht beeinflusst. Um diese Frage aber endgültig entscheiden zu können, wäre eine placebokontrollierte Studie notwendig, die idealerweise Placebo mit Fresh Frozen Plasma und Prothrombinkomplex-Konzentrat vergleichen würde.

\footnotetext{
Referenz

1. Hart RG et al. Stroke 2012; 43: $1511-7$
} 